\title{
Theory of a Single Dye Molecule Blinking with a Diffusion-Based Power Law Distribution
}

\author{
Wei-Chen Chen and R. A. Marcus* \\ Noyes Laboratory, 127-72 California Institute of Technology, Pasadena, California 91125, United States
}

ABSTRACT: In single molecule studies of injection of an electron from a photoexcited dye into a semiconductor nanoparticle or into a film of such nanoparticles, the injection may be into the conduction band or into the band gap, depending on the system. The theory of the process and its return are discussed, in particular when a power law for the waiting time distribution may be expected and what that power might be. To this end a reaction-diffusion equation is set up and solved. When the injection is into the conduction band, a power law is predicted for the return of the electron to the dye cation but not for the injection. After a short time, the law for the waiting time distribution has a power of -1 . At short times, before the slower return due to an increasing radius is recognized, the power law is $-1 / 2$. When the injection is into the band gap, a -1 power law is predicted for both the injection and the return. Available data are discussed in terms of the theory. A corollary is that single molecule studies for the injection can determine whether the injection is into the band gap or into the conduction band. The theory is tested by single molecule studies of various systems, such as comparing different dye $-\mathrm{TiO}_{2}, \mathrm{dye}-\mathrm{Al}_{2} \mathrm{O}_{3}$, and dye $-\mathrm{ZrO}_{2}$ systems and comparing specific dye- $\mathrm{TiO}_{2}$ systems as a function of $\mathrm{pH}$, and dye hole injection into p-type $\mathrm{NiO}$.

\section{INTRODUCTION}

In dye sensitized solar cells (DSSC) the initial process of photoexcitation of a dye molecule is usually followed by the injection of the excited electron from the dye into a film of sintered or nonsintered semiconductor nanoparticles. ${ }^{1}$ The electron injection is in competition with both the fluorescence of the excited dye and the radiationless transition to the electronic ground state of the dye. ${ }^{2-5}$ The electron injected into a nanoparticles then either diffuses to an electrode that is part of an electrical circuit or undergoes an energy-wasteful step, such as reacting with an oxidant in solution or recombining with the dye cation.

Recently there have been single molecule studies of the fluorescence intermittency of a single photoexcited dye molecule on a semiconductor nanoparticle system or on a crystal surface. ${ }^{6-10}$ The switch between light and dark periods appears to be of two types: (1) a system that has "on" and "off" periods, i.e., has fluorescent and nonfluorescent periods, and (2) a system that is also "partially on" and "partially off". We consider systems of the first type. The second type is more complicated in that there may be more than two states or the "binning" of the data may have included more than one "on" period within a bin.

In this first type of system a power law for the survival probability has been observed for the "dark" (the nonfluorescent) phase ${ }^{9-13}$ and sometimes for the "light" (fluorescent) phase ${ }^{11,13}$ of the dye molecule. For the dark to light transition at least two different mechanisms are proposed in the literature to explain a power law for the transition: (1) distribution of tunneling barriers for the ejected electron to return to the dye cation from a distribution of trap depths ${ }^{14-17}$ or (2) a diffusion of the ejected electron in the semiconductor to return to the dye cation. ${ }^{18}$ In any case more detailed information on the injection/recombination steps in the DSSC should be obtainable from single molecular studies. The above two classes of mechanisms have also been proposed previously as alternative interpretations of the intermittent fluorescence ("blinking") of quantum dots. ${ }^{14-17,19-21}$

In the present article on the initial steps in a DSSC we describe the solution of a time-dependent reaction-diffusion theory prompted by the approximate $t^{-1}$ power law. Here, the electron injected from the dye into the semiconductor can diffuse in an assembly of nanoparticles, perhaps via trap-to-trap tunneling. ${ }^{17}$ It can return to the dye cation with a timedependent probability that depends on how far it has diffused.

The present model suggests two different types of timebehavior for the on to off periods, according to the energy of the injected electron relative to the band gap. (1) If an excited electron has an energy high enough for it to be injected into the conduction band, the injection is followed by a trap-to-trap diffusion back to the dye cation. A power law for the survival probability density in the dark state is expected, but not for the injection itself, i.e., not for the light state. (2) If instead, for a very wide band gap semiconductor such as $\mathrm{Al}_{2} \mathrm{O}_{3}$ or $\mathrm{ZrO}_{2}$, where the electron is to be injected into the band gap, an acceptor trap can diffuse and ultimately, if close enough, accept the electron from the excited dye, the theory predicts that the survival probability density of both the dark state and the light state obeys a power law. The theory can also be applied to systems in which there is hole injection from the dye, rather than electron injection.

This electron injection into the band gap may proceed via surface states that typically exist in the band gap. When the energetics of the photoexcited dye relative to the conduction band edge are not known, the single molecule intermittency provides, according to this suggestion, a tool for determining whether injection is into the band gap or into the conduction band and can yield information on injection rate constants.

Received: April 20, 2012

Published: June 11, 2012 
Analysis of the fluorescence intermittency may also provide insight into initial steps in DSSC, namely, on the injection and geminate recombination. In DSSC both geminate and nongeminate recombination can in principle occur.

This reaction-diffusion mechanism provides a specific prediction of the actual power in the power law. For an alternative theory containing a distribution of rate constants, perhaps for tunneling from different trap depths, the power is an adjustable parameter. There may be systems where one interpretation is appropriate and others where the reverse is true. In any case, for either interpretation both single molecule and ensemble data are needed in a detailed mechanistic study. It was found that single molecule and ensemble studies for quantum dots, for example, provided complementary information. ${ }^{19,20,22}$ The diffusion constant $D$ inferred from the present interpretation of the recombination data may be compared with the $D$ measured in ensembles for trap-to-trap surface diffusion. $^{23,24}$

In the present article the focus is on specific single molecule experiments, the predictions, and the comparison with the experimental data. A reaction-diffusion model is set up for the injection and recombination steps for dye-on-surface systems and compared with the experimental data. There is a body of related literature on geminate recombination of the electron ejected from a photoexcited dye, extensive references being given in ref 25, largely for 1- and 3-dimensional systems, rather than for the present 2-dimensional system.

The article is organized as follows: In section 2 the kinetics scheme and the relevant differential equation is described and solved by a Laplace transformation. An analytic approximation to the latter yields the observed power law. In section 3 a numerical solution is given that covers all time regions. The results are compared with experiment in section 4. For an important part of the time domain, presently for which observations have been made in single molecule systems, the analytical solution has a simple form. Comparison of the predicted power in the power law for two classes of singlemolecule experiments is also given and discussed. An analysis is given that distinguishes the two different regions of injection: injection into the conduction band and injection into the band gap. Concluding remarks are given in section 5 .

\section{THEORY}

2.1. Power Law for the Dark State. When a single dye molecule on a semiconductor nanoparticle film or on a crystalline surface is photoexcited, the subsequent processes include the relaxation to its ground state via radiative and nonradiative transitions, injection of the excited electron into the semiconductor surface, and recombination with the dye cations. When the dye cation is, as is common, nonfluorescent, the dye molecule becomes dark until an electron recombines with it, and then the dye resumes its fluorescence under the steady illumination. The recombination with the dye cation is, in a solar cell, in competition with diffusion of the electron to an electrode, the reduction of the cation with an reductant in solution, and unwanted electron transfer from nanoparticles to the oxidant in solution. We consider in this paper the injection and the return of the electron to the dye molecule cation, an example of germinate recombination.

The dye molecule on a surface is approximated by a small disk with a radius $r_{0}$ on a two-dimensional surface. The injected electron diffuses on the surface and is able to return to the dye molecule cation with a total flux $J$ at $r_{0}$. The diffusion equation for the electron probability density function in the dark $\rho$ and the boundary conditions are given by

$$
\begin{aligned}
& \frac{\partial \rho}{\partial t}=D \frac{1}{r} \frac{\partial}{\partial r}\left(r \frac{\partial \rho}{\partial r}\right) \quad\left(r \geq r_{0}\right) \\
& \left.J\right|_{r=r_{0}}=-\left.2 \pi r D \frac{\partial \rho}{\partial r}\right|_{r=r_{0}}=-2 \pi r_{0} k \rho\left(r=r_{0}, t\right) \\
& \rho(r) \rightarrow 0 \quad \text { as } \quad r \rightarrow \infty
\end{aligned}
$$

where $D$ is the diffusion coefficient of the electron on the surface in this dark period. At first we neglect the Coulombic attraction between the electron and the dye cation, and include it later in section 3.2 to see its effect. We also neglect long-range electron transfer at a distance in this initial treatment. In eq $2 k$ is the rate constant of the back electron transfer from the film to the molecule cation, in units of length/time, common units in electrochemical kinetics for electron transfer at a surface.

Before injection $(t<0)$ the photoexcited electron is bound within the dye molecule, $r<r_{0}$. We denote by $\mathcal{P}(t)$, the probability that the electron is outside the dye molecule $(r \geq$ $\left.r_{0}\right) . \mathcal{P}(t)$ behaves as a unit step function, the step occurring at the instant of injection of the electron into the surface. During the instant after the injection, before the electron has time to diffuse away or to transfer back to the dye cation, the probability density function $\rho(r, t)$ of the injected electron on the film is concentrated at the circumference of the molecule cation, $r=r_{0}^{+}$, and so at the periphery of a region $2 \pi r_{0}^{+}$. Due to the assumption of isotropy on the surface, the initial distribution function $\rho(r, t \rightarrow 0)$ depends only on $r$ and $t$, and so the survival probability $\mathcal{P}$ at time $t$ is

$$
1=\mathcal{P}\left(t \rightarrow 0^{+}\right)=\int_{r_{0}}^{\infty} \mathrm{d} r 2 \pi r \rho\left(r, t \rightarrow 0^{+}\right)
$$

Thus, at time infinitesimally greater than $t=0$, before it has time to diffuse, it is like a delta function confined to $r=r_{0}$ and so is written as

$$
2 \pi r \rho\left(r, 0^{+}\right)=\delta^{+}\left(r-r_{0}\right)
$$

where $\delta^{+}$is like the Dirac delta function but for the interval $(0, \infty)$, instead of $(-\infty, \infty)$ in the variable $r$, i.e., $\int_{r 0}^{\infty} \mathrm{d} r \delta^{+}\left(r-r_{0}\right)$ $=1$. Examples of the development of $\rho(r, t)$ with time are shown in Figure 1.

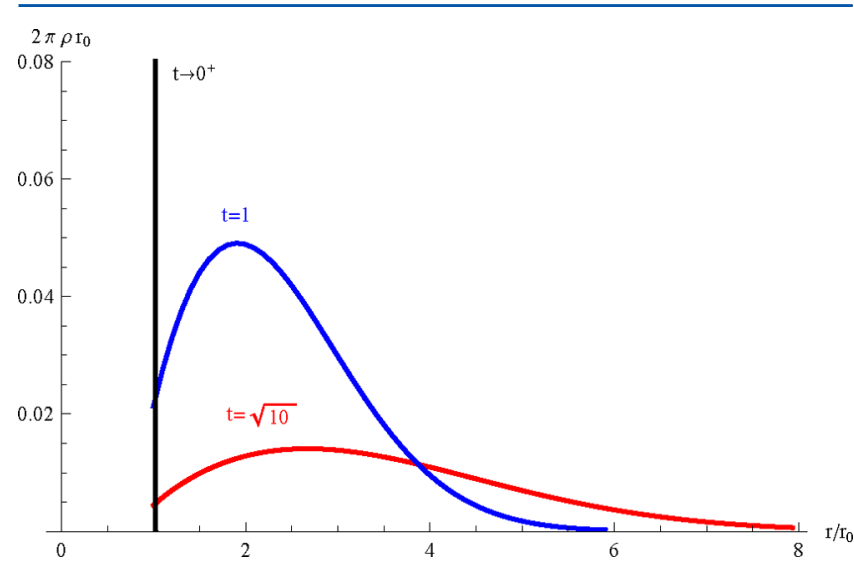

Figure 1. Development of $\rho(r, t)$ with time. The $k$ and $D$ values used in the plot are $1 r_{0}$ time ${ }^{-1}$ and $1 r_{0}^{2}$.time ${ }^{-1}$, respectively. For any other $D$, $D^{\prime}$, one rescales the time axis, as $D^{\prime} t / D$. 
Applying the above initial condition and applying the Laplace transform, i.e., $\mathcal{L}\{F(t)\}=\tilde{F}(s)$ to eqs 1 and 2, we have

$$
\begin{aligned}
& \frac{\partial^{2} \tilde{\rho}}{\partial u^{2}}+\frac{1}{u} \frac{\partial \tilde{\rho}}{\partial u}-\tilde{\rho}=-\frac{\delta^{+}\left(u-u_{0}\right)}{2 \pi D u} \\
& \left.D \frac{\partial \tilde{\rho}}{\partial u}\right|_{u=u_{0}}=k \sqrt{D / s} \tilde{\rho}\left(u_{0}, s\right)
\end{aligned}
$$

where $u$ and $u_{0}$ are introduced to simplify the notation. They denote $r(s / D)^{1 / 2}$ and $r_{0}(s / D)^{1 / 2}$, respectively, $s$ being the transform variable. The solution of this differential equation is

$$
\tilde{\rho}(r, s)=\frac{1}{2 \pi u_{0}} \frac{K_{0}(u)}{D K_{1}\left(u_{0}\right)+k \sqrt{D / s} K_{0}\left(u_{0}\right)}
$$

where $K_{n}$ is the $n$th order modified Bessel function of the second kind. ${ }^{26}$

The desired quantity for the intermittent fluorescence is the derivative of the time-dependent survival probability density $p(t),{ }^{19}$

$$
p(t)=-\mathrm{d} \mathcal{P}(t) / \mathrm{d} t=-\int_{r_{0}}^{\infty} 2 \pi r \mathrm{~d} r \partial \rho(r, t) / \partial t
$$

Its Laplace transform is

$$
\tilde{p}(s)=1-s \int_{r_{0}}^{\infty} 2 \pi r \mathrm{~d} r \tilde{\rho}=\frac{k K_{0}\left(u_{0}\right)}{\sqrt{s D} K_{1}\left(u_{0}\right)+k K_{0}\left(u_{0}\right)}
$$

To gain some insight into the time behavior, we consider first the short time approximation, i.e., the behavior when the Laplace transform-related variable $u_{0}$ is large. Equation 10 then gives

$$
\begin{aligned}
\tilde{p}(s) & \approx \frac{r_{0} k}{D} \frac{K_{0}\left(u_{0}\right)}{u_{0} K_{1}\left(u_{0}\right)} \\
& =\frac{r_{0} k}{D}\left(I_{0}\left(u_{0}\right) K_{0}\left(u_{0}\right)+\frac{I_{1}\left(u_{0}\right) K_{0}^{2}\left(u_{0}\right)}{K_{1}\left(u_{0}\right)}\right) \\
& \approx \frac{r_{0} k}{D}\left(I_{0}\left(u_{0}\right) K_{0}\left(u_{0}\right)+I_{1}\left(u_{0}\right) K_{1}\left(u_{0}\right)\right)
\end{aligned}
$$

The property that $K_{0}\left(u_{0}\right) / K_{1}\left(u_{0}\right)$ approaches unity when $u_{0} \rightarrow$ $\infty$ was used in deriving the above approximations.

The inverse transform of the expression in eq 13 is given by

$$
\begin{aligned}
p(t) & \approx \frac{r_{0} k}{2 D t} \exp \left(-\frac{r_{0}^{2}}{2 D t}\right)\left(I_{0}\left(\frac{r_{0}^{2}}{2 D t}\right)+I_{1}\left(\frac{r_{0}^{2}}{2 D t}\right)\right) \\
& \approx \begin{cases}\frac{k}{\sqrt{\pi D}} t^{-1 / 2} & t \ll \frac{r_{0}^{2}}{2 D} \\
\frac{r_{0} k}{2 D} t^{-1} & t \gg \frac{r_{0}^{2}}{2 D}\end{cases}
\end{aligned}
$$

Accordingly, the approximate derivative of the survival probability density $p(t)$ of the dark state obeys a $-1 / 2$ and -1 power law at shorter and longer times, respectively. The approximate eq 15 is discontinuous at $t=r_{0}{ }^{2} / 2 D$ but the more general eq 14 , is not.

The experimental diffusion constant $D$ of an electron in a $\mathrm{TiO}_{2}$ nanoparticle film is in the range of $10^{-3}-10^{-7} \mathrm{~cm}^{2} \mathrm{~s}^{-1}$, depending on temperature, the electron concentration, nanoparticle size, and whether or not the nanoparticle film is sintered. ${ }^{23,24,27-29}$ Possible mechanisms for such a large range of diffusion coefficients in ensemble measurements are noted later. The radius of a dye molecule is around $1 \mathrm{~nm}$. On this basis the $-1 / 2$ power law of the survival probability would only occur at times no longer than $50 \mathrm{~ns}$, and not observable for the usual time scale of milliseconds to seconds in single molecule experiments for studying the survival probability in the fluorescent intermittency.

2.2. Power Law for the Light State: Injection into the Band Gap. When the electron acceptor (a trap) is in the band gap, as in $\mathrm{Al}_{2} \mathrm{O}_{3}$ or $\mathrm{ZrO}_{2}$ that has a very wide band gap, the trap concentration is small, but the trap can diffuse. It can therefore reach and accept an electron from the photoexcited dye molecule, causing the dye to become dark. Therefore, by assuming that $\rho$ is the hole probability density function on the surface, $k$ is the rate constant of the electron transfer from the dye to the hole in the band gap, and $D$ is the diffusion coefficient of the hole on the surface in the light period, we can extend the results obtained in the previous section to explain the light state behavior when the dye injects photoexcited electron into the band gap.

\section{NUMERICAL RESULTS}

3.1. Inverse Laplace Transform. By introducing values of $k, D$, and $r_{0}$, the survival probability derivative, $-\mathrm{d} P(t) / \mathrm{d} t$, for the dark state can be obtained by a numerical inverse Laplace transformation of eq 10. The result is given in Figure 2. The

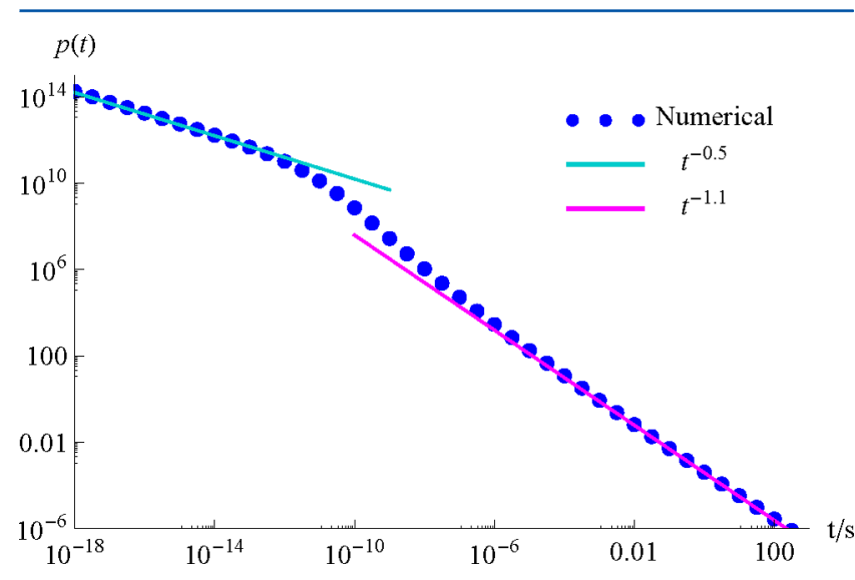

Figure 2. Survival probability density obtained by the numerical evaluation of the inverse Laplace transform of eq 10 . The $k, D$, and $r_{0}$ values used in the plot are $10^{10} \mathrm{~nm} \cdot \mathrm{s}^{-1}, 10^{-5} \mathrm{~cm}^{2} \cdot \mathrm{s}^{-1}$, and $1 \mathrm{~nm}$, respectively. For any other $D, D^{\prime}$, one rescales the time axis, as $D^{\prime} t / D$.

power law exponent for the short time regime is about -0.5 for a wide range of diffusion coefficients and is given by the approximate value in eq 15 . Several ensemble measurements show that the back-electron-transfer rate from colloidal $\mathrm{TiO}_{2}$ nanoparticles to dye cations has a distributed kinetics that is from a few picoseconds to nanoseconds. ${ }^{30,31}$ The half-life of the absorption recovery of dye molecules in the Weng et al. ensemble experiment is around $50 \mathrm{ps.}^{31}$ The radius of the dye molecule is around $1 \mathrm{~nm}$. Assuming the $k$ value is around the dye size divided by the half-life ( $50 \mathrm{ps}$ ), then $k$ is about $2 \times 10^{10}$ $\mathrm{nm} \cdot \mathrm{s}^{-1}$. When the diffusion coefficient is near the lower bound in ensemble measurements, $10^{-7} \mathrm{~cm}^{2} \mathrm{~s}^{-1}$, the numerical power law changes to -1.15 at long times, a value slightly larger than 
the approximate value of -1 given by eq 15 . When $D$ is near the upper bound, $10^{-3} \mathrm{~cm}^{2} \cdot \mathrm{s}^{-1}$, in the ensemble measurements, the numerical slope in this time region is -1.02 . The numerical power law at very long times has little dependence on the diffusion coefficient, $D$.

3.2. Effect of a Coulombic Field on the Recombination. Any Coulombic force between the ionized molecule and the trapped electron would be expected to reduce further the diffusion range of the trapped electron, and thereby to shorten the duration time of the dark state. The diffusion equation with a drift potential is a Smoluchowski equation,

$$
\frac{\partial \rho}{\partial t}=\nabla \cdot\left(D \nabla \rho+\frac{D}{k_{\mathrm{B}} T} \rho \nabla U\right) \quad\left(r \geq r_{0}\right)
$$

where $k_{\mathrm{B}}$ is the Boltzmann constant, $T$ is the absolute temperature, and $U$ is the Coulombic potential of the dye cation and the electron on the surface. Its boundary and initial conditions are the same as eqs 2,3 , and 5 . The dielectric constant of $\mathrm{TiO}_{2}\left(\varepsilon_{\mathrm{r}}=85\right)^{32}$ is used to calculate the potential, $U(r)$. A numerical simulation of reaction-diffusion in a Coulombic interaction confirms that the recovery ratio for the encapsulated molecule was found to be $>98 \%$ in $100 \mathrm{~s}$, which is slightly larger than a $\sim 96 \%$ recovery for reactiondiffusion without the Coulombic interaction. The survival probability density of the cation in the reaction-diffusion system in a Coulombic field still has a -1.1 exponent, as seen in Figure 3, so the Coulombic field has little effect on the power law behavior for the observed time scale in the current singlemolecule experiments.

\section{DISCUSSION}

4.1. Comparison with Experiment: Single Dye Molecule on a $\mathrm{TiO}_{2}$ Nanoparticle Film. Recently, Wu et al. studied the blinking of single rhodamine-type dye molecules spin-coated on a $\mathrm{TiO}_{2}$ nanoparticle film. ${ }^{9}$ A power law was reported for the dark state disappearance. They also noted that

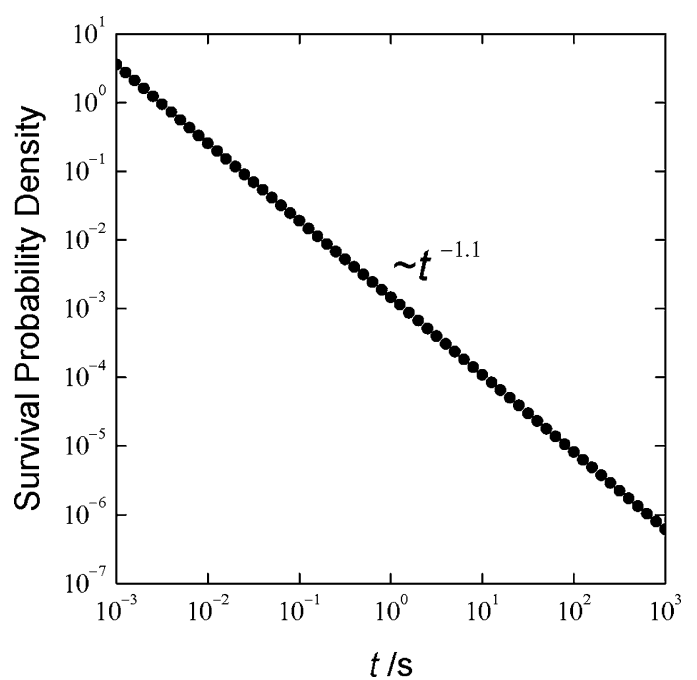

Figure 3. Dark-state distribution obtained by numerically propagating a diffusion equation that includes the Coulombic force between the day cation and the ejected electron. In the simulation the parameters used are $k=2 \times 10^{8} \mathrm{~nm} \cdot \mathrm{s}^{-1}, D=10^{-6} \mathrm{~cm}^{2} \cdot \mathrm{s}^{-1}, r_{0}=1 \mathrm{~nm}$, and 85 for the dielectric constant of $\mathrm{TiO}_{2} \cdot{ }^{32}$ The simulated exponent of the power law is about -1.1 . For any other $D, D^{\prime}$, one rescales the time axis, as $D^{\prime} t / D$. the probability distribution for the light state did not follow a single exponential decay and did not follow a power law. Singlemolecule results for the observed probability distribution for the dark state were given for a dye molecule with and without an encapsulation of the dye molecule, as in Figure 4a,b, respectively. Several ensemble measurements show that the electron transfer in dye-sensitized colloidal $\mathrm{TiO}_{2}$ nanoparticles can be fitted in a multiexponential function, and its average half-life distribution is from a few picoseconds to nanoseconds. ${ }^{30,31}$ The diameter of the dye molecule is around $2 \mathrm{~nm}$. In the present study, the transfer rate constant for recombination with the dye molecule cation on the film is assumed from the ensemble data to be $2 \times 10^{10} \mathrm{~nm} \cdot \mathrm{s}^{-1}$ for a bare dye molecule, which is comparable with the product of the estimated rate constant by $\mathrm{Wu}$ et al. ${ }^{9}$ and the size of a dye molecule. Because the attenuation factor $\beta$ due to the tunneling effect in electron transfer at dye-sensitized nanocrystalline $\mathrm{TiO}_{2}$ films is $\sim 1 \AA^{-1}, 33$ and the half-height of the capsulate molecule is $\sim 4.6 \AA$, the estimated back-electron-transfer rate for encapsulated molecule for the encapsulated dye is reduced to $2 \times 10^{8} \mathrm{~nm} \cdot \mathrm{s}^{-1} \cdot{ }^{34}$

The experimental electron diffusion coefficients in nonsintered $\mathrm{TiO}_{2}$ nanoparticles are around $4 \times 10^{-7}$ to $4 \times 10^{-6}$ $\mathrm{cm}^{2} \cdot \mathrm{s}^{-1}, 28$ which is lower than the sintered ones by $\sim 2$ orders of magnitude at similar photoexcited electron density on the nanoparticle film. ${ }^{29}$ Because the spin-coated nanoparticle film without the sintering process is used in the $\mathrm{Wu}$ et al. experiment, ${ }^{9}$ the electron diffusion coefficient in the current simulation is assumed to be $10^{-6} \mathrm{~cm}^{2} \cdot \mathrm{s}^{-1}$. In a typical observation time $(\sim 100 \mathrm{~ms})$, the electron would then travel over 100 nonsintered nanoparticles, so providing a basis for the continuum approach used in the present diffusion-reaction model. The diameter of each nanoparticle is $10-5 \mathrm{~nm}$.

For $D=10^{-6} \mathrm{~cm}^{2} \cdot \mathrm{s}^{-1}$ our numerical results for both the encapsulated and bare dye molecules in their dark-state distributions give about a -1.1 exponent for the survival probability density. The plots of the survival probability density are shown as the solid lines in Figure 4. The present model is in reasonable agreement with the observation for the encapsulated molecule in the observed time region, as seen in Figure 4a. The present model assumes the same rate constant of the electron transfer for all single molecules on the nanoparticle film. However, $\mathrm{Wu}$ et al. found a distribution of fluorescence halflifes of single bare dye molecules on a $\mathrm{TiO}_{2}$ nanoparticle film, ${ }^{9}$ a distribution that may be due to various orientations of the bare dye molecules on the nanoparticles. If so, this effect also yields a distribution of rate constants for electron transfer for the bare molecule, which has not been included in the present analysis and could cause a deviation from the simple theory at long times.

The observed longest duration of a continuous dark state in the present single molecule experiment is less than $100 \mathrm{s.}^{9}$ Application of the final value theorem ${ }^{35}$ for the probability in the Laplace space gives its time domain behavior as time approaches infinity,

$$
\lim _{t \rightarrow \infty} \mathcal{P}(t)=\lim _{s \rightarrow 0} s \mathcal{P}(s)=\lim _{s \rightarrow 0} s \int 2 \pi r \mathrm{~d} r \tilde{\rho}=0
$$

This result for the current diffusion model thus leads to there being a full recovery of the photoionized dye molecules in the very long time limit. In the current numerical results the recovery at $100 \mathrm{~s}$ is $\sim 100 \%$ and $\sim 96 \%$ for bare and encapsulated molecules, respectively. 


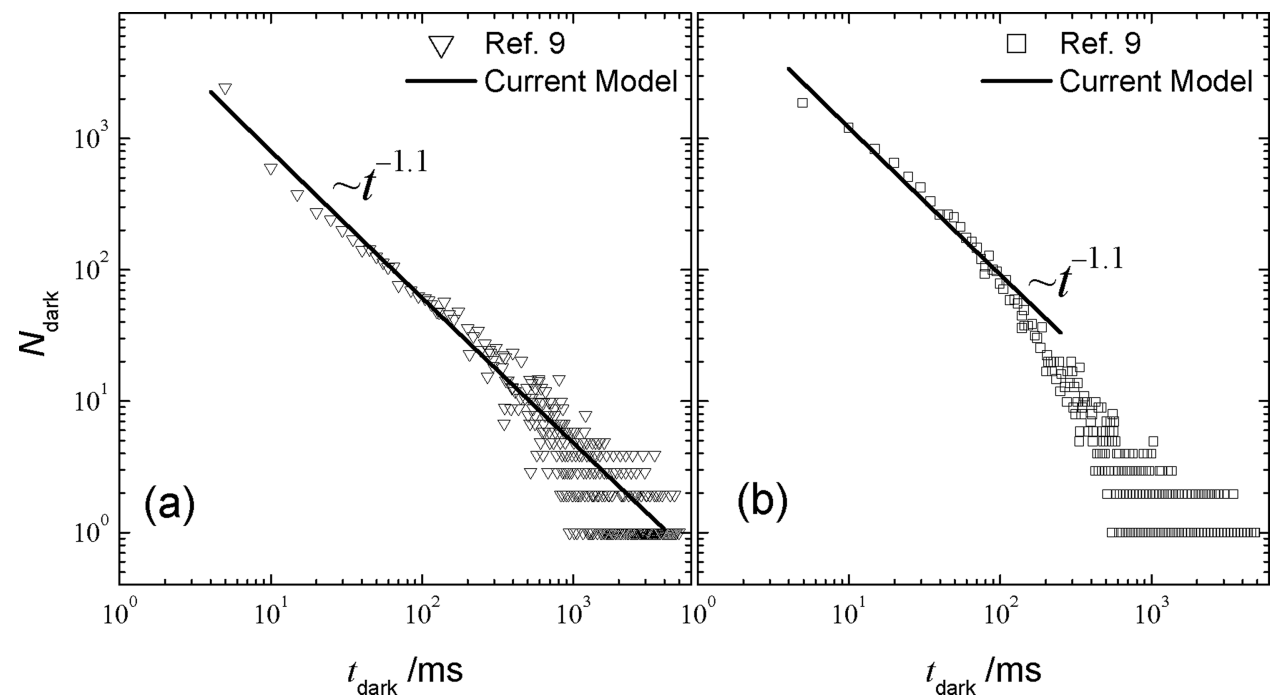

Figure 4. Dark-state distributions. The open symbols are measured dark state distributions of single dye molecules by Wu et al. ${ }^{9}$ Open triangles (a) and squares (b) refer to the single dye molecule with and without an encapsulation, respectively. The encapsulation reduces the back-electrontransfer rate, $k$, which are $2 \times 10^{8}$ and $2 \times 10^{10} \mathrm{~nm} \cdot \mathrm{s}^{-1}$ in (a) and (b), respectively. ${ }^{9,34}$ The line is obtained by the current model with the following parameters: $D=10^{-6} \mathrm{~cm}^{2} \cdot \mathrm{s}^{-1}$, and $r_{0}=1 \mathrm{~nm}$. The calculated exponents of the power law are around -1.1 .

4.2. Electron Injection into an $\mathrm{Al}_{2} \mathrm{O}_{3}$ Band Gap. In contrast with the experiment $\mathrm{Wu}$ et al., where a power law was found only for the dark state of a single dye molecule on a $\mathrm{TiO}_{2}$ nanoparticle surface, ${ }^{9}$ Schirra et al. observed a power law behavior for both the light and the dark states for a single dye on a $\mathrm{Al}_{2} \mathrm{O}_{3}$ crystalline surface. ${ }^{11}$ The experimental band gaps for $\mathrm{TiO}_{2}{ }^{36}$ and $\mathrm{Al}_{2} \mathrm{O}_{3}{ }^{37,38}$ are $\sim 3.0$ and $\geq 8.5 \mathrm{eV}$, respectively. Reflecting the relatively small band gap in $\mathrm{TiO}_{2}$ and the excited state energy of the dye molecule, the free-energy change $\Delta G^{0}$ for electron transfer from the excited state of the dye molecule to the bottom of conduction band of the $\mathrm{TiO}_{2}$ is quite negative, $-0.46 \mathrm{eV} .^{9}$ Due to the exothermic reaction and the high density of electron acceptors in the conduction band, the half-life distribution of the light state of the dye is for direct injection into the conduction band. Because there are a large number of "acceptors" for the injected electron (empty conduction band states), the transfer of the excited electron from a dye molecule into the conduction band has a half-life less than $100 \mathrm{ps},{ }^{9}$ instead of requiring a slow diffusion of the electron-acceptor traps on the $\mathrm{TiO}_{2}$ nanoparticle film to capture the injected electron.

In contrast, because of the large band gap the photoexcited electron of the dye molecule on the $\mathrm{Al}_{2} \mathrm{O}_{3}$ surface does not have enough energy to be injected into the conduction band. Accordingly, the neutral dye molecule may require many photoexcitations and fluorescence before the electron-trap on the surface has diffused to the dye molecule and then traps the photoexcited electron and causes the dye to form a cation and hence become dark. In general, to have experimentally a power law behavior for the light state, the model in this article requires that the energy of the excited state is less than the edge of the conduction band. Measurements on this expectation, especially for dye molecules having a smaller excitation energy $(<1.5 \mathrm{eV})$ on the $\mathrm{TiO}_{2}$ nanoparticle film, would permit a further test of the model.

The energetic scheme of the injection into the conduction band for dye- $-\mathrm{TiO}_{2}$ or into the band gap for dye $-\mathrm{ZrO}_{2}$ is shown in Figure 5. Examples of the dyes studied include alizarin, ${ }^{39}$ quinizarin, ${ }^{40}$ and 7 -hydroxycoumarin 4 -acetic acid. ${ }^{41}$

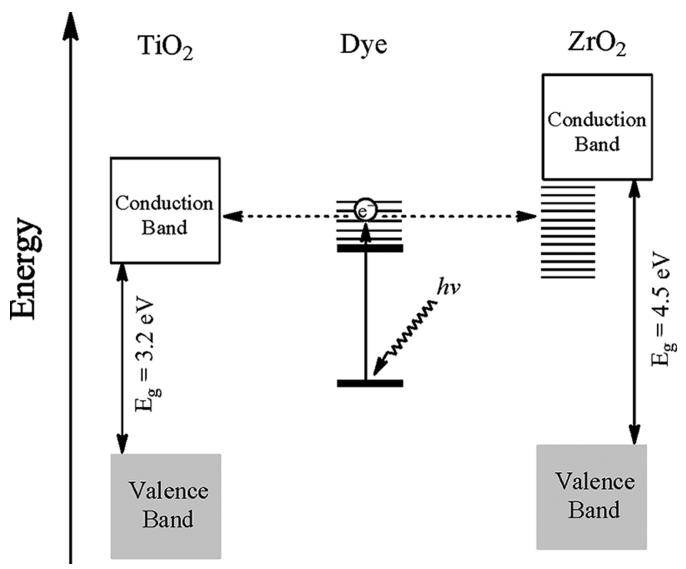

Figure 5. Energetic scheme of the dye- $\mathrm{TiO}_{2}$ and dye $-\mathrm{ZrO}_{2}$ system. The dashed arrows show the injection of a photoexcited electron into the conduction band of $\mathrm{TiO}_{2}$ nanoparticles or into the states in the band gap of $\mathrm{ZrO}_{2}$ nanoparticles if the excited-state energy of the dye molecule is between 3.2 and $4.5 \mathrm{eV}$. Examples of dye molecules include alizarin, ${ }^{39}$ quinizarin, ${ }^{40}$ and 7 -hydroxycoumarin 4 -acetic acid. ${ }^{41}$

All of these dyes have a photoexcited state between 3.2 and 4.5 $\mathrm{eV}$. One would thus anticipate, on the basis of current theory, that in a single molecule experiment the injection into $\mathrm{TiO}_{2}$ would have a power law only for the return to the light state, whereas it should have a power law for both the formation and the disappearance of the dark state in the case of $\mathrm{ZrO}_{2}$, under the condition where in the former the injection is into the conduction band and in the latter into the band gap.

The conduction band edge position of $\mathrm{TiO}_{2}$ nanoparticles depends on its ambient $\mathrm{pH}$ value. Matylitsky et al. observed that the photoexcited electron of the alizarin $-\mathrm{TiO}_{2}$ system injects an electron into the conduction band and into the band gap at $\mathrm{pH} 2$ and $\mathrm{pH} 9$, respectively. ${ }^{42}$ If only the position of the conduction and valence bands is the determining factor that a power law is observed, then the prediction from the present theory is that a power law would be observed only in the light state at a lower $\mathrm{pH}$ environment, and in both light and dark states at higher $\mathrm{pH}$. 


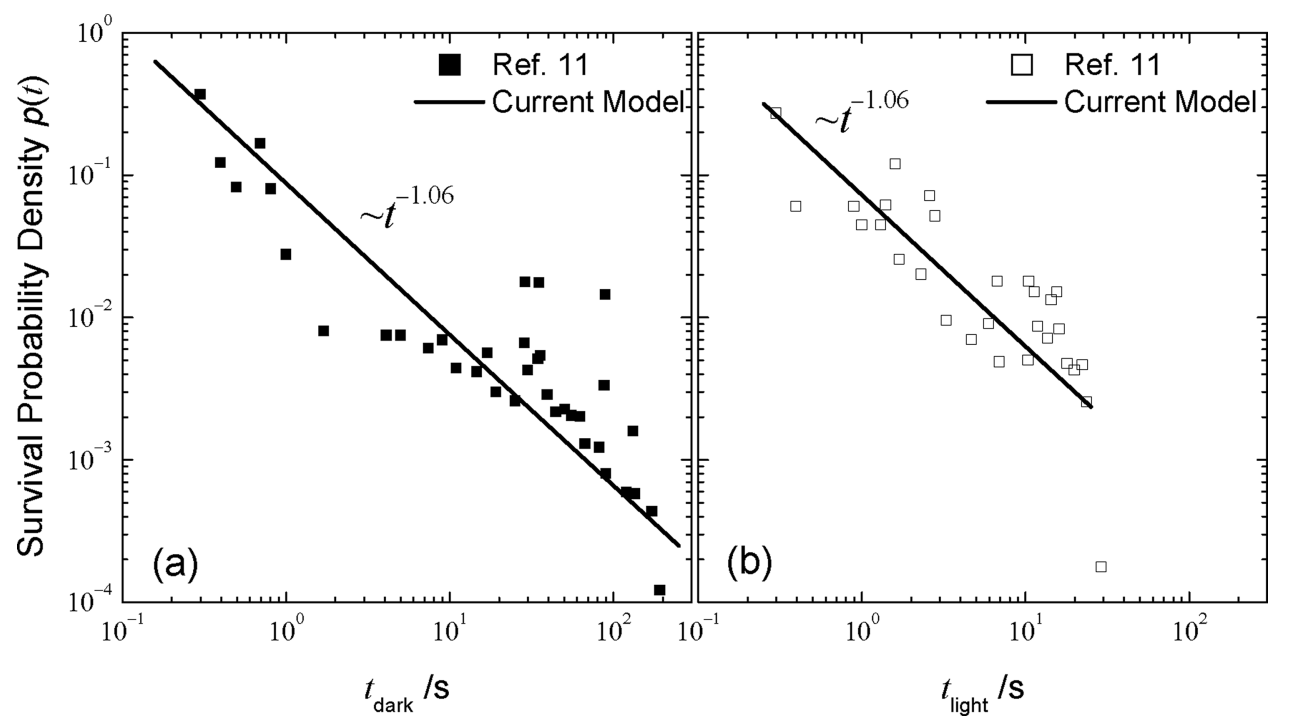

Figure 6. Dark-state (a) and light-state (b) distributions of survival probability densities. The squares are the experimental data of Schirra et al. ${ }^{11}$ The diffusion constant, not available in the literature, is assumed to be $10^{-3} \mathrm{~cm}^{2} \cdot \mathrm{s}^{-1}$ for both electrons and traps on the crystal surface states. The lines show the calculated power laws of the current model with the parameters: $k=2 \times 10^{10} \mathrm{~nm} \cdot \mathrm{s}^{-1}$ and $r_{0}=1 \mathrm{~nm}$. The calculated exponents of the power law for dark- and light-state distributions in the reaction-diffusion model are -1.06 .

4.3. Comparison with Experiment: Single Dye Molecules on an $\mathrm{Al}_{2} \mathrm{O}_{3}$ Crystalline Surface. The survival probability for the fluorescence intermittency of single molecules deposited on a crystalline $\mathrm{Al}_{2} \mathrm{O}_{3}$ (0001) surface was studied by Schirra et al. ${ }^{11}$ Both dark- and light-state distributions showed power law behavior, as in Figure 6a,b, respectively. A model with a distribution of activated charge transfer and recombination kinetics was suggested to explain the results. ${ }^{11}$ Their data indicate that power law behavior is observed even under conditions with minimum disorder on the surface. The system may not be compatible, therefore, with the disordered matrix model that has been proposed. ${ }^{15,43}$ The diffusion model, on the other hand, is consistent with the observation of power laws found for both the light and dark state in this system. In the present model, the diffusion considered is on the surface; i.e., it involves surface states.

Guo et al. calculated the surface states of an $\mathrm{Al}_{2} \mathrm{O}_{3}$ (0001), the crystal face terminated with an $\mathrm{Al}$ layer. They predicted the existence of surface states at 2 and $8 \mathrm{eV}$ below the conduction band minimum. ${ }^{44}$ (The band gap is $\geq 8.5 \mathrm{eV}$.) ${ }^{37,38}$ By combining several surface analysis methods and analyzing the energy-loss spectra, Gillet and Ealet identified the surface states of $\mathrm{Al}_{2} \mathrm{O}_{3}$ monocrystal (0001) surface and located two energy states, 3.4 and $5.7 \mathrm{eV}$ below the conduction band minimum. ${ }^{45}$ Such surface states may be a source of the present traps postulated for electron on the surface for the recombination and for the hole diffusion on the surface accepting the injected electron.

If the photoexcited electron from the neutral dye molecule is injected into a localized surface state near the surface/molecule interface, and then diffuses among the surface states before returning and neutralizing the molecule, the current reactiondiffusion model can describe this experiment. A diffusion constant for this particular system does not appear to have been reported in the literature. However, as noted earlier, the present model gives a power law in the survival probability distribution for a wide range of diffusion constants. By introducing some $D$, $k$, and $r_{0}$ values in eq 10 (as those in the caption of Figure 6) and then applying a numerical inverse Laplace transform, we obtain a survival probability rate, $-\mathrm{d} \mathcal{P}(t) / \mathrm{d} t$, with a -1.06 power law in the range of observations, as seen in Figure 6a. The value of -1.06 gives a somewhat better agreement with the observations for the time range than a steeper power law of -1.16 fitted by Schirra et al., ${ }^{19}$ but the difference is small.

As noted in the previous section, the photoexcited electron of the single dye molecule on the $\mathrm{Al}_{2} \mathrm{O}_{3}$ surface is injected into the surface trap states in the band gap in the present theory. By applying the reaction-diffusion model for the light state, we show a numerical power law result in Figure $6 \mathrm{~b}$, obtained by introducing assumed the $D, k$, and $r_{0}$ values in eq 10 , and then applying a numerical inverse Laplace transform. The model gives the power law of the on-time in the observed time range with the numerical power law around -1.06 . It gives a good agreement with the experimental results of Monti and coworkers. ${ }^{11}$

4.4. Electron vs Hole Injection into the Band Gap. The photoexcited electron injected into an empty trap that diffuses on the surface is assumed above in deriving the behavior of the light state. The electron injection and diffusion mechanism corresponds to the n-type DSSC. ${ }^{46}$ For a p-type DSSC, ${ }^{13,46}$ the switching from the light to dark state of the photoexcited may be that a photoinduced hole in the dye molecule is injected into a hole trap in the band gap on the surface. The above results for both light and dark states would still be the same even when the above "donor" type of the single dye and the "acceptor" type of the surface are changed. Bian et al. studied the blinking of single dye molecules on p-type $\mathrm{NiO}$ nanoparticle surfaces with and without insulating $\mathrm{Al}_{2} \mathrm{O}_{3}$ layers and found that the survival probability density for both light and dark state have a power law with both the "on" and "off" values of the power centered around -1 and also showing a near exponential tail. ${ }^{13}$ So, the theory for the intermittent fluorescence in n-type systems applies also for hole injection from a dye into a p-type semiconductor. For brevity of presentation, only the case of electron injection from a dye molecule is given above.

4.5. Diffusion Coefficients. It was observed experimentally in ensemble experiments that the diffusion coefficient depends on incident light intensity, nanoparticle size, and temperature 
and in particular on the concentration of photoinduced electron-dye cation pairs in the $\mathrm{TiO}_{2}$ nanoparticle film. $^{23,24,27-29}$ As noted above, the observed coefficients are in the vicinity of $10^{-3}-10^{-7} \mathrm{~cm}^{2} \cdot \mathrm{s}^{-1}$, where sintered nanoparticles have a larger diffusion coefficient than nonsintered. One explanation of the dependence is that deeper traps are more likely filled when the photoinduced electron density is higher, so enhancing the diffusion coefficient. ${ }^{47,48}$ The increased electron density changes the Fermi level of the $\mathrm{TiO}_{2}$ so as to make the traps in the band gap closer to the conduction band. ${ }^{49}$ Alternatively, there is a percolation effect depending on the density of excited dye molecules, and the light intensity, which affects the ratio of geminate to nongeminate recombination. In terms of a percolation effect of the light intensity mentioned above, the laser intensity dependence of the diffusion coefficients may be due to enhanced migration by th electron moving to some ionized center different from its parent one. More dye molecules become ionized at higher laser intensity, enhancing the effect. Although the accurate diffusion coefficient is currently not available in the single molecule experiment, the numerical result of the current diffusion model shows that the power law behavior is quite robust over a wide range of diffusion coefficients.

A time- and position-independent diffusion coefficient is used in the present reaction-diffusion equation. It assumes that only the surface states within a narrow energy distribution have significant contribution for diffusion. This assumption is very different from the models with a distribution of traps. ${ }^{14-17}$ Further experimental studies on the particular of the $\sim-1$ power law can help clarify this issue. Finally, we comment on the predicted change of slope at short times from $-1 / 2$ to -1 at longer times. The power law of $-1 / 2$ is common for 1 dimentional reaction-diffusion systems at short times, whereas the additional $-1 / 2$ power in the present case is due to the increase in $r$ with time, as the effect of the decreased rate of return becomes smaller because of an enhanced area $\pi r^{2}$ occupied by the diffusing particle, compared with this quantity $\pi r_{0}^{2}$ at $t=0$.

\section{CONCLUSIONS}

A reaction-diffusion mechanism is proposed to explain the power law distribution of fluorescence intermittency of single dye molecules on the nanoparticle film and on a single crystal. The time-dependent reaction-diffusion equation gives specific powers for the distribution. By applying the Laplace transform as well as numerical approaches, we can derive the approximate power law observed in the experiment. Analytically, one obtains $a-1$ power law in the long time region and $a-1 / 2$ power law at short times, the latter during the $2 \mathrm{D}$ effect is still negligible. The latter power may be difficult to be observed in current single molecule experiments due to its short time region of its validity. Similar to the analytical result, the numerical result also gives two distinct power laws, $\sim-1 / 2$ and $\sim-1$, in the short and long time regions, respectively. In the long time region, the model numerically gives a fairly robust power law, -1.02 to -1.15 , in a very wide range of diffusion constants. In an alternative mechanism, the power in the power law has been treated as an adjustable parameter.

The current model predicts that the energy of the conduction band being higher than that of the excited state of the dye molecule is essential for experimentally observing the power law survival probability density in the light state and can be tested for different dye-semiconductor combinations.

\section{AUTHOR INFORMATION}

\section{Corresponding Author}

*E-mail: ram@caltech.edu.

\section{Notes}

The authors declare no competing financial interest.

\section{ACKNOWLEDGMENTS}

It is a pleasure to acknowledge the support of this research by the National Science Foundation, the Office of Naval Research, and the Army Research Office. We thank Prof. M. E. MichelBeyerle of Nanyang Technological University (NTU) for helpful comments. The present work has been performed in part during the tenure of RAM as Nanyang Visiting Professor at the NTU. W.-C.C. particularly wishes to acknowledge the support from a fellowship from NTU.

\section{REFERENCES}

(1) O’Regan, B.; Grätzel, M. Nature 1991, 353, 737-740.

(2) Grätzel, M. Acc. Chem. Res. 2009, 42, 1788-1798.

(3) O'Regan, B. C.; Durrant, J. R. Acc. Chem. Res. 2009, 42, 17991808.

(4) Peter, L. Acc. Chem. Res. 2009, 42, 1839-1847.

(5) Hagfeldt, A.; Gratzel, M. Acc. Chem. Res. 2000, 33, 269-277.

(6) Wang, Y.; Wang, X.; Lu, H. P. J. Am. Chem. Soc. 2009, 131, 9020-9025.

(7) Biju, V.; Micic, M.; Hu, D. H.; Lu, H. P. J. Am. Chem. Soc. 2004, 126, 9374-9381.

(8) Holman, M. W.; Liu, R. C.; Adams, D. M. J. Am. Chem. Soc. 2003, $125,12649-12654$

(9) Wu, X.; Bell, T. D. M.; Yeow, E. K. L. Angew Chem. Int. Ed. 2009, $48,7379-7382$.

(10) Wang, Y.; Wang, X.; Ghosh, S. K.; Lu, H. P. J. Am. Chem. Soc. 2009, 131, 1479-1487.

(11) Schirra, L. K.; Tackett, B. S.; Blumenfeld, M. L.; Monti, O. L. A. J. Chem. Phys. 2009, 131, 124702.

(12) Bott, E. D.; Riley, E. A.; Kahr, B.; Reid, P. J. J. Phys. Chem. A 2010, 114, 7331-7337.

(13) Bian, Z.; Tachikawa, T.; Cui, S.-C.; Fujitsuka, M.; Majima, T. Chem. Sci. 2012, 3, 370-379.

(14) Kuno, M.; Fromm, D.; Johnson, S. T.; Gallagher, A.; Nesbitt, D. J. Phys. Rev. B 2003, 67, 125304.

(15) Cichos, F.; von Borczyskowski, C.; Orrit, M. Curr. Opin. Colloid Interface Sci. 2007, 12, 272-284.

(16) Wustholz, K. L.; Bott, E. D.; Isborn, C. M.; Li, X.; Kahr, B.; Reid, P. J. J. Phys. Chem. C 2007, 111, 9146-9156.

(17) Nelson, J.; Haque, S. A.; Klug, D. R.; Durrant, J. R. Phys. Rev. B 2001, 63, 205321.

(18) Nelson, J.; Chandler, R. E. Coord. Chem. Rev. 2004, 248, 11811194.

(19) Tang, J.; Marcus, R. A. J. Chem. Phys. 2005, 123, 054704.

(20) Tang, J.; Marcus, R. A. J. Chem. Phys. 2005, 123, 204511.

(21) Jung, Y. J.; Barkai, E.; Silbey, R. J. Chem. Phys. 2002, 284, 181194.

(22) Tang, J.; Marcus, R. A. J. Chem. Phys. 2006, 125, 044703.

(23) Kopidakis, N.; Neale, N. R.; Zhu, K.; van de Lagemaat, J.; Frank, A. J. Appl. Phys. Lett. 2005, 87, 202106.

(24) Zhu, K.; Kopidakis, N.; Neale, N. R.; van de Lagemaat, J.; Frank, A. J. J. Phys. Chem. B 2006, 110, 25174-25180.

(25) Agmon, N. Phys. Chem. Chem. Phys. 2011, 13, 16548-16557.

(26) Abramowitz, M., Stegun, I. A., Eds. Handbook of Mathematical Functions, with Formulas, Graphs, and Mathematical Tables; U.S. Government Printing Office: Washington, DC, 1972; pp 374- 378.

(27) Nakade, S.; Saito, Y.; Kubo, W.; Kitamura, T.; Wada, Y.; Yanagida, S. J. Phys. Chem. B 2003, 107, 8607-8611. 
(28) Kopidakis, N.; Benkstein, K. D.; van de Lagemaat, J.; Frank, A. J.; Yuan, Q.; Schiff, E. A. Phys. Rev. B 2006, 73, 045326.

(29) Mori, S.; Sunahara, K.; Fukai, Y.; Kanzaki, Y.; Wada, Taisukeand; Yanagida, S. J. Phys. Chem. C 2008, 112, 20505-20509.

(30) Ramakrishna, G.; Ghosh, H. N.; Singh, A. K.; Palit, D. K.; Mittal, J. P. J. Phys. Chem. B 2001, 105, 12786-12796.

(31) Weng, Y. X.; Wang, Y. Q.; Asbury, J. B.; Ghosh, H. N.; Lian, T. Q. J. Phys. Chem. B 2000, 104, 93-104.

(32) http://www.azom.com/article.aspx?ArticleID=1179.

(33) Clifford, J. N.; Palomares, E.; Nazeeruddin, M. K.; Gratzel, M.; Nelson, J.; Li, X.; Long, N. J.; Durrant, J. R. J. Am. Chem. Soc. 2004, 126, 5225-5233.

(34) The height of the encapsulated molecule is $9.1 \AA$. Wu et al. estimated the back-electron-transfer rate by using the full height in the exponent of the exponential term for the dependence of reaction rates as electron tunneling distance. ${ }^{9}$ We note that if the dye molecule is fully encapsulated and at the center of the encapsule molecule, the distance from the dye to the $\mathrm{TiO}_{2}$ nanoparticles should be around half of the height, $4.55 \AA$, and the rate of the encapsulated molecule is larger than that estimated by $\mathrm{Wu}$ et al. ${ }^{9}$ by $\sim 2$ orders of magnitude.

(35) Franklin, G. F.; Powell, J. D.; Emami-Naeini, A. Feedback Control of Dynamic Systems; Pearson Prentice Hall: Englewood Cliffs, NJ, 2006; pp 90-92.

(36) Cronemeyer, D. C. Phys. Rev. 1952, 87, 876-886.

(37) Abramov, V. N.; Karin, M. G.; Kuznetsov, A. I.; Sidorin, K. K. Sov. Phys. Solid State 1979, 21, 80-86.

(38) Olivier, J.; Poirier, R. Surf. Sci. 1981, 105, 347-356.

(39) Huber, R.; Sporlein, S.; Moser, J. E.; Gratzel, M.; Wachtveitl, J. J.

Phys. Chem. B 2000, 104, 8995-9003.

(40) Ramakrishna, G.; Singh, A. K.; Palit, D. K.; Ghosh, H. N. J. Phys. Chem. B 2004, 108, 4775-4783.

(41) Ramakrishna, G.; Singh, A. K.; Palit, D. K.; Ghosh, H. N. J. Phys. Chem. B 2004, 108, 12489-12496.

(42) Matylitsky, V. V.; Lenz, M. O.; Wachtveitl, J. J. Phys. Chem. B 2006, 110, 8372-8379.

(43) Schuster, J.; Cichos, F.; von Borczyskowski, C. Appl. Phys. Lett. 2005, 87.

(44) Guo, J.; Ellis, D. E.; Lam, D. J. Phys. Rev. B 1992, 45, 1364713656.

(45) Gillet, E.; Ealet, B. Surf. Sci. 1992, 273, 427-436.

(46) Hagfeldt, A.; Boschloo, G.; Sun, L.; Kloo, L.; Pettersson, H. Chem. Rev. 2010, 110, 6595-6663.

(47) Bisquert, J. J. Phys. Chem. B 2004, 108, 2323-2332.

(48) Bisquert, J. J. Phys. Chem. C 2007, 111, 17163-17168.

(49) Bisquert, J.; Vikhrenko, V. S. J. Phys. Chem. B 2004, 108, 23132322. 